\title{
Mice Deficient in Glycerol-3-Phosphate Acyltransferase-1 Have a Reduced Susceptibility to Liver Cancer
}

\author{
Jessica M. Ellis ${ }^{1,2}$, David S. Paul ${ }^{1}$, Michael A. Depetrillo ${ }^{1}$, Bhanu P. Singh ${ }^{3,4}$, David E. \\ Malarkey $^{3}$, and Rosalind A. Coleman ${ }^{1}$ \\ ${ }^{1}$ Department of Nutrition, University of North Carolina, Chapel Hill, North Carolina, USA \\ ${ }^{2}$ Department of Biological Chemistry, Center for Metabolism and Obesity Research, Johns \\ Hopkins University School of Medicine, Baltimore, Maryland, USA \\ ${ }^{3}$ National Toxicology Program, National Institute of Environmental Health and Sciences, Cellular \\ and Molecular Pathology Branch, Research Triangle Park, North Carolina, USA \\ ${ }^{4}$ Dupont Haskell Global Centers for Health and Environmental Sciences, Newark, Delaware, USA
}

\section{Abstract}

The risk of hepatocellular carcinoma increases with the persistence of non-alcoholic fatty liver disease. Triacylglycerol synthesis is initiated by glycerol-3-phosphate acyltransferase (GPAT). Of four isoforms, GPAT1 contributes 30-50\% of total liver GPAT activity, and we hypothesized that it might influence liver susceptibility to tumorigenesis. C57B1/6 mice deficient in GPAT1 were backcrossed 6 times to $\mathrm{C} 3 \mathrm{H}$ mice. After exposure to the carcinogen diethylnitrosamine (DEN) and the tumor promoter phenobarbital, male Gpat1 $1^{-/-}$mice, compared with controls ( ppat $^{+/+}$), had 93\% fewer macroscopically visible nodules per liver at 21 weeks of age and 39\% fewer at 34 weeks of age. Microscopically, control mice had increased numbers of foci of altered hepatocytes, particularly the basophilic subtype, as well as more, and malignant, liver neoplasms than did the Gpat $^{-1-}$ mice. At 21 weeks of age, 50\% (4/8) of control mice (50\%) had hepatocellular adenomas with an average multiplicity (tumors per tumor-bearing-animal) of 4.3, while none occurred in 8 Gpat $^{-1-}$ mice. At 34 weeks of age, all 15 control mice (100\%) had hepatocellular adenomas with an average multiplicity of 5.2 compared to an incidence of $93 \%$ in $\mathrm{Gpat}^{-1-}$ mice and multiplicity of 3.1. HCCs were observed in $13 \%$ of control mice and in only $6 \%$ of $\mathrm{Gpat1}^{-/-}$mice. These data show that alterations in the formation of complex lipids catalyzed by Gpat1 reduce susceptibility to DEN-induced liver tumorigenesis.

\section{Keywords}

hepatocellular carcinoma; glycerolipid synthesis; non-alcoholic fatty liver disease; non-alcoholic steatohepatitis; diethylnitrosamine

\section{Introduction}

Hepatocellular carcinoma (HCC) is the third leading cause of cancer-related death worldwide, accounting for 600,000 deaths annually (World Health Organization [WHO] 2009; Parkin et al. 2005). During the past two decades, the incidence of HCC in developed

Copyright (C) 2011 by The Author(s)

Address correspondence to: Rosalind A. Coleman, Department of Nutrition, CB\# 7461, University of North Carolina, Chapel Hill, NC 27599, USA; rcoleman@unc.edu. 
countries has increased due to both the increased prevalence of infection with the hepatitis C virus (El-Serag and Mason 1999; Bosch et al. 2004) and the growing numbers of obese individuals. It has been predicted that the major causes for liver transplantation in the next 20 years will be HCC and cirrhosis resulting from non-alcoholic fatty liver disease (NAFLD; Pillai and Rinella 2009; Schreuder et al. 2008). Non-alcoholic fatty liver disease is defined as the accumulation of intracellular triacylglycerol (TAG) in the liver of individuals who consume less than $20 \mathrm{~g}$ of alcohol/day (Neuschwander-Tetri and Caldwell 2003). The spectrum of NAFLD ranges from benign steatosis to non-alcoholic steatohepatitis (NASH). Non-alcoholic steatohepatitis with significant amounts of inflammation and fibrosis can progress to cirrhosis, hepatic failure, and HCC.

Fatty liver, particularly in obese individuals, requires the action of the metabolic pathway of triacylglycerol (TAG) biosynthesis initiated by the acylation of glycerol-3-phosphate at the $s n-1$ position by glycerol-3-phosphate acyltransferase (GPAT; Coleman and Lee 2004). GPAT is the rate limiting step in the de novo synthesis of both glycerophospholipids and TAG (Kennedy 1961; Coleman and Lee 2004). In mammals, this reaction is catalyzed by one of 4 GPAT isoforms (GPAT1-4), each encoded by a separate gene. GPAT isoforms vary in their tissue expression and subcellular location. GPAT1, which is located in the outer mitochondrial membrane, is most prominent in adipose tissue, kidney, and liver. The role of GPAT1 in liver is of particular interest because of its high contribution (30-50\%) to total hepatic GPAT activity compared with other tissues ( 10\%; Hammond et al. 2002) and the up-regulation of its expression by SREBP1c, a transcription factor regulated by insulin. Thus, hepatic GPAT1 mRNA and activity are high under physiological conditions when an obesogenic diet and high insulin concentrations promote TAG synthesis. Glycerol-3phosphate acyltransferase 1 competes with carnitine palmitoyltransferase-1 (CPT-1) for acyl-CoAs, thereby promoting their incorporation into glycerolipids while limiting their entry into the mitochondria where they would be oxidized (Hammond et al. 2005). This conclusion is supported by the observation that after Gpat $^{-/-}$mice are fed a high-fat, highsucrose diet for 4 months, they have higher amounts of serum $\beta$-hydroxybutyrate and hepatic acyl-carnitines, aswell as reduced hepatic content of diacylglycerol and TAG. The importance of GPAT1 for hepatic TAG accumulation is supported by the $60 \%$ lower hepatic TAG content in mice lacking both GPAT1 and leptin (ob/ob-Gpat1 ${ }^{-1-}$ mice) when compared to mice that are deficient in leptin alone (ob/ob; Wendel et al. 2010).

Mouse strains are differentially susceptible to HCC (Diwan, Rice, and Ward 1990). In a strain comparison study, the carcinogen DEN initiated tumor formation in all $\mathrm{C} 3 \mathrm{H}$ mice but in only $30 \%$ of $\mathrm{C} 57 \mathrm{Bl} / 6$ mice, and the average number of foci of altered hepatocytes per liver was 20- to 50-fold greater in $\mathrm{C} 3 \mathrm{H}$ mice compared with $\mathrm{C} 57 \mathrm{Bl} / 6$ mice (Drinkwater and Ginsler 1986). In a comparison of spontaneous tumors and those induced with vinyl carbamate, the prevalence of hepatocellular adenomas varied from $2.4-14.1 \%$ for spontaneous tumors and between $22.8-72.9 \%$ in a low-dose group with the C57B1/6 strain being least affected and the $\mathrm{C} 3 \mathrm{H}$ strain among the most severely affected (Takahashi et al. 2002). Furthermore, pulmonary metastases were frequently present in $\mathrm{C} 3 \mathrm{H}$ mice. Because mice on the C57B1/6 strain are resistant to the induction of HCC (Diwan, Rice, and Ward 1990), we backcrossed Gpat1 $^{-1-}$ and control mice to the liver tumor-susceptible C3H strain.

We have reported that a deficiency of GPAT1 in liver results in increased rates of fatty acid oxidation, a 20\% increase in the rate of reactive oxygen species (ROS) production, an

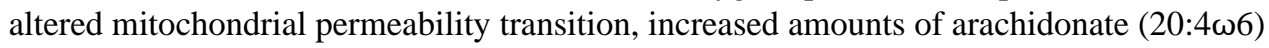
in the $s n-2$ position of phosphatidylcholine and phosphatidylethanolamine, and higher amounts of the arachidonate peroxidation product, 4-hydroxynonenal in liver. The increased oxidative stress is associated with balanced increases in both hepatocyte apoptosis and proliferation (Hammond et al. 2007). Obesity has been associated with HCC (Park et al. 
2010) and transformed cells may develop resistance to apoptosis (Schattenberg,

Schuchmann, and Galle 2011). Because the content of TAG is low in livers from Gpat1-1mice and cell turnover is high, we hypothesized that the tumorigenic potential of DENinduced liver tumorigenesis in $\mathrm{Gpat1}^{-/-}$mice would be reduced compared to wildtype.

\section{Methods}

\section{Animal Treatment}

Gpat1 $^{-1-}$ mice (C57B1/6 background) were backcrossed to the HCC-susceptible C3H/ HeNCrl strain (Diwan, Rice, and Ward 1990; C3H; Charles River Laboratories) for six

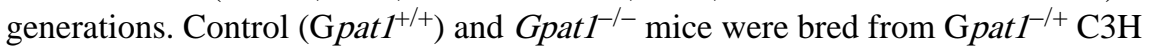
backcrossed mice and were housed in a pathogen-free barrier facility (12 h light/dark cycle) in accordance with the UNC IACUC and allowed free access to water and food (Prolab RMH 3000 SP76 chow). Mouse body weights were measured weekly. Vehicle or diethylnitrosamine (DEN, Sigma) dissolved in PBS was injected intraperitoneally into 15.5day-old control and Gpat $^{-1-}$ male pups at a dose of $4 \mathrm{mg} / \mathrm{kg}$ body weight. Phenobarbital was administered ad libitum in drinking water at 500 parts per million from the age of 5 weeks to necropsy. Tissues were harvested at 8,21, and 34 weeks of age $(n=10-15)$. Individual tumors, liver, and lungs were snap frozen and stored at $-80^{\circ} \mathrm{C}$.

\section{Liver Evaluation}

At necropsy of 21- and 34-week-old mice, all surfaces of livers and lungs were examined for macroscopically visible nodules. The number of nodules was recorded for each lobe of the liver and lung. Whole lung, nodules greater than $5 \mathrm{~mm}$, and one representative section of the left, median, and caudate liver lobes was cut for histological evaluation, fixed in fresh $4 \%$ paraformaldehyde-PBS for $24 \mathrm{~h}$, dehydrated in 70\% ethanol, and routinely processed and embedded in paraffin. Paraffin embedded tissue was cut into 5- $\mu \mathrm{m}$ sections and stained with hematoxylin and eosin. Microscopically proliferative and nonproliferative liver lesions were evaluated by two pathologists (BS and DM) without knowledge of genetic status. Foci of altered hepatocytes (FAH) were classified as either basophilic, eosinophilic, clear cell, or mixed; and hepatocellular adenomas (HCA) and carcinomas (HCC) were classified using previously published criteria (Harada et al. 1999). Briefly, FAH, which are considered putative preneoplastic lesions (Harada et al. 1999), were identified as groups of hepatocytes with altered tinctorial properties of the cytoplasm that range in size from less than a lobule to sometimes greater than four lobules, with no or minimal compression of surrounding tissues. Foci of altered hepatocytes often merge imperceptibly with surrounding parenchyma. Mixed FAH are composed of $<80 \%$ of one cell type. Hepatocellular adenomas consisted of expansile and compressive masses of neoplastic hepatocytes, most often without portal triads, that sometimes contained peripheral regions with varying numbers of trabeculae, notmore than two cells wide. When trabeculae of neoplastic hepatocytes with three cells or more were found, together with cellular pleomorphism and/or other malignant features, the tumor was classified as an HCC.

\section{Enzyme Activity}

Livers were minced on ice and then homogenized with 10 up-and-down strokes by a motordriven Teflon-glass homogenizer in a buffer containing $10 \mathrm{mM}$ Tris- $\mathrm{HCl}$ (pH 7.4), $250 \mathrm{mM}$ sucrose, $1 \mathrm{mM}$ dithiothreitol, and $1 \mathrm{mM}$ EDTA. The total membrane fraction was isolated by centrifuging at $100,000 \times \mathrm{g}$ for $1 \mathrm{~h}$ at $4^{\circ} \mathrm{C}$. Protein concentration was determined by the bicinchoninic acid method (Pierce, Rockford, IL) with bovine serum albumin as the standard. GPAT activity was assayed for $10 \mathrm{~min}$ at $25^{\circ} \mathrm{C}$ in a $200-\mu l$ reaction mix containing $75 \mathrm{mM}$ Tris- $\mathrm{HCl}$, pH 7.5, $4 \mathrm{mM} \mathrm{MgCl} 2,1 \mathrm{mg} / \mathrm{ml}$ bovine serum albumin, $1 \mathrm{mM}$ dithiothreitol, $8 \mathrm{mM} \mathrm{NaF}, 800 \mu \mathrm{M}\left[{ }^{3} \mathrm{H}\right]$ glycerol-3-phosphate, and $80 \mu \mathrm{M}$ palmitoyl-CoA in 
the presence or absence of $2 \mathrm{mM} N$-ethylmaleimide (NEM). For NEM inhibition studies, membrane protein was incubated in $1 \mathrm{mM} \mathrm{NEM}$ on ice for $15 \mathrm{~min}$ before its addition to the reaction mixture. The NEM inhibition of GPAT2, 3, and 4 isoforms has been reported previously (Lewin et al. 2004; Cao et al. 2006; Nagle et al. 2008; Chen et al. 2008). Each reaction was initiated with $15-30 \mu \mathrm{g}$ of protein and was proportional to the amount of time and protein used. $\left[{ }^{3} \mathrm{H}\right]$ glycerol-3-phosphate was synthesized enzymatically from $\left[2-{ }^{3} \mathrm{H}\right]$ glycerol (1 mCi/ml; Perkin-Elmer Life Sciences; Chang and Kennedy 1967).

\section{RNA Quantification}

Total RNA was isolated from liver with the Qiagen RNeasy Micro Tissues Kit. cDNA was synthesized (Applied Biosystems High Capacity cDNA RT Kit), and the reaction performed using SYBR Green (Applied Biosystems) detection with primers specific to the gene of interest with equal amounts of cDNA (10 ng/reaction), and detected using a Taq-man thermo-cycler (BioRad). Results were normalized to the housekeeping gene Gapdh and expressed as arbitrary units of $2-\Delta \mathrm{CT}$.

\section{Cell Proliferation}

Bromodeoxyuridine (BrdU) in the drinking water $(0.2 \mathrm{~g} / \mathrm{L})$ was incorporated into proliferating hepatocytes for $48 \mathrm{~h}$ before necropsy. Paraffin-embedded liver sections were hydrolyzed in $4 \mathrm{~N} \mathrm{HCl}$ and permeabilized in $0.2 \%$ pepsin (Dako) in $0.2 \mathrm{~N} \mathrm{HCl}$ solution. Immunostaining was performed with a monoclonal antibody against BrdU (Dako) diluted 1:200 in 1\% bovine serum albumin in PBS. Immunohistochemical detection was performed using a horseradish peroxidase-conjugated secondary antibody followed by development with a 3,3'-diaminobenzidine chromogen solution (Dako). Slides were counterstained using Mayer's hematoxylin (Sigma). Ten BrdU-stained images from the left lobe of each liver were randomly selected for analysis using Image Pro 7.0 software (Media Cybernetics). A labeling index was calculated from at least 2,000 cells per liver as the number of positively stained nuclei/total number of nuclei counted.

\section{PPARa-Mediated Proliferative Effects}

To determine whether the lipogenic pathway involving GPAT1 activity is critical for PPARa-mediated proliferative effects, control and Fpat $^{-1-}$ mice on the C57B1/6 background were fed the PPARa ligand Wy-14,643 (Sigma) (Gonzalez and Shah 2008) in their drinking water at $500 \mathrm{ppm}$ for 1 week before tissue harvest $(n=3)$.

\section{Statistical Analysis}

Data are expressed as mean \pm SEM. Lesion incidences were compared between control and Gpat $^{-1-}$ mice using Fisher's exact test, and lesion multiplicity was compared using the Mann-Whitney test. Statistical comparisons of all other endpoints between control and Gpat $1^{-1-}$ mice were determined using unpaired, two-tailed, Student's $t$-tests; $p$-values $\leq .05$ were considered significant.

\section{Results \\ GPAT Expression Profiles of Gpat1 ${ }^{-1-}$ Mice on Both the C57BI/6 and C3H Strain Backgrounds Were Similar}

Gpat $1 \mathrm{mRNA}$ abundance was similar in control liver from C3H and C57Bl/6 strains (Figure 1A). Compared with controls, Gpat1 mRNA abundance in $G p a t 1^{-1-}$ liver was reduced 80$90 \%$, regardless of strain background. The mRNA expression of Gpat2, Gpat3, and Gpat4 did not increase in $\mathrm{C} 3 \mathrm{H}$ Gpat $^{-1-}$ livers, demonstrating lack of compensation by the three other GPAT isoforms (Figure 1B). The activities of GPAT2, 3, and 4, but not GPAT1, are 
inhibited by sulfhydryl reagents like NEM. NEM-sensitive GPAT activity in the liver was similar in control and Fpat $^{-1-}$ mice, whereas NEM-resistant GPAT activity (GPAT1 activity) was $85 \%$ lower than in control mice (Figure 1C). Thus, the extent of Gpat1 knockout in the liver was similar in mice on the $\mathrm{C} 57 \mathrm{BL} / 6$ and the $\mathrm{C} 3 \mathrm{H}$ strain backgrounds.

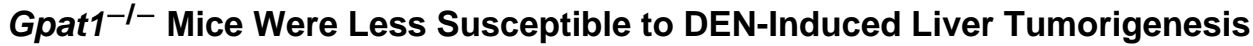

Tumorigenesis was initiated by treating $\mathrm{Gpat}^{-1-}$ and control mice on the $\mathrm{C} 3 \mathrm{H}$ background with a single injection of the mutagenic carcinogen, DEN, on day 15 of age. Tumorigenesis was then promoted with phenobarbital supplied in the drinking water until the mice were 21 or 34 weeks old. Weight gain did not differ between the genotypes (data not shown). At necropsy, macroscopically visible, tan nodules present on the surface of the liver and lungs were quantified. Compared with controls treated with DEN and phenobarbital, the average number of macroscopic foci per liver was $93 \%$ lower in Fpat $^{-1-}$ mice at 21 weeks of age and $39 \%$ lower at 34 weeks of age (Figure 2A). The average number of macroscopic foci in control and ppat $^{-1-}$ mice per liver was 27 versus 2 foci and 65 versus 40 foci at 21 and 34 weeks of age, respectively. Of the four liver lobes, the left and median lobes contained the largest number of visible macroscopic foci in both genotypes. At 34 weeks of age compared to control mice, a reduction in Gpat $^{-1-}$ liver foci was noted for the left (58\%), median $(37 \%)$, and right (58\%) lobes (Figure 2B).

DEN treatment primarily affects liver tissue, but it can also induce primary proliferative lesions (hyperplasias and/or neoplasms) in lung (Reznik-Schuller 1983). Overall, DEN treatment induced relatively few macroscopic lung foci. The average number of macroscopic foci observed per lung in control and Gpat $^{-1-}$ mice was 0 versus 0.09 foci and 1 versus 0.87 foci at 21 and 34 weeks of age, respectively. No differences between genotypes were found either in the macroscopic incidence or the average number of foci per lung between wildtype and $\mathrm{Gpat1}^{-1-}$ mice (data not shown). Microscopically, a few of the lesions were identified as either focal alveolar epithelial (type II pneumocyte) hyperplasia or alveolar/ bronchiolar adenomas. The lack of genotype difference in the development of lung foci is consistent with the relatively low activity of GPAT1 (mitochondrial GPAT) in lung (Schlossman and Bell 1977; Das and Haldar 1987).

Microscopic evaluation of representative liver sections from control and $\mathrm{Gpat}^{-1-}$ mice supported the overall decrease in hepatocellular proliferative lesions (FAH and neoplasms) in Gpat $^{-1-}$ mice. Four types of foci were identified (Table 1). In both genotypes the majority of the examined liver foci at 21 weeks were basophilic (Figure 5 and Table 1). Basophilic foci are highly proliferative and associated with the development of HCC in rats fed the PPARa ligand Wy-14,643 (Marsman and Popp 1994). At 34 weeks, themajority of foci evaluated were of mixed type. Gpat1 $1^{-/}$mice at 21 weeks had developed fewer foci overall $(p<.05)$, decreased basophilic and eosinophilic foci, but little or no difference in the number of mixed and clear foci. Similarly, at $34 \mathrm{wks}$, Fpat $^{1--}$ mice had developed fewer basophilic foci $(p<.05)$ and decreases in eosinophilic and mixed foci. In contrast, Gpat $1^{-1-}$ mice developed a greater number of clear foci than did control mice $(p<.05)$.Clear cell FAH are often found to have increased cytoplasmic glycogen with or without lipid, indicating possible dysfunctional cellular metabolism.

Representative liver sections from control and $\mathrm{Gpat1}^{-{ }^{--}}$mice were evaluated microscopically for the incidence of primary HCC and hepatocellular adenomas in livers and for lung metastases (Table 1). At 21 weeks, 17 adenomas were detected in 50\%of control mice (multiplicity $=4.3$ ), whereas no adenomas were present in the Gpat $^{-1-}$ mice (Table 1, $p<.05$ ). At 34 weeks, adenomas were present in 100\% of control mice and in $93 \%$ of Gpat $^{-1-}$ mice, whereas HCCs were observed in $13 \%$ of control mice and in only $6 \%$ of Gpat $^{-1-}$ mice. Tumor multiplicity (the number of liver neoplasms per tumor-bearing 
animals) was similar in both groups. No tumor metastases were found in representative lung sections of either genotype.

\section{Rates of Hepatocyte Proliferation Were Higher in Gpat1-/- Mice}

Our data demonstrate that ppat $^{-1-}$ mice on the $\mathrm{C} 3 \mathrm{H}$ back-ground were less susceptible to DEN-induced liver tumorigenesis. We previously reported that Gat $^{-/-}$mice on the C57Bl/ 6 background have increases in both apoptosis and proliferation, suggesting that the increased rate of hepatocyte turnover might result in a lower susceptibility to liver cancer (Hammond et al. 2007). We examined the rate of cell proliferation in 8 week old Gpat $^{-1-}$ mice to verify that the increased rates of proliferation observed in pat $^{-1-}$ mice on the $\mathrm{C} 57 \mathrm{Bl} / 6$ background were preserved in the $\mathrm{C} 3 \mathrm{H}$ background. Compared to controls, the rate of cell proliferation, assessed by BrdU incorporation into hepatocytes during a $48 \mathrm{~h}$ exposure, was approximately 2-fold higher in Gpat $^{-1-}$ livers (Figure 3), although liver weights were unchanged. These data suggest that increased hepatocyte turnover in Gpat ${ }^{-1-}$ livers reduced the survival of aberrant, tumorigenic cells.

\section{GPAT1 Was Not Critical for PPARa-Mediated Induction of Hepatocellular Proliferation}

PPARa increases the numbers of both peroxisomes and mitochondria and upregulates mitochondrial genes involved in lipid metabolism (Bogacka et al. 2005). Because supplementation with the PPARa agonist Wy-14,643 promotes hepatocellular proliferation and can promote cancer by decreasing the expression of the microRNA let-7c gene, thereby allowing proliferative c-myc to remain active (Gonzalez and Shah 2008), we asked whether GPAT1 was required for the ability of PPARa to increase the rate of hepatocyte proliferation. Wy-14,643 treatment increased liver weight, as a percentage of body weight, by 2.4-fold in control mice and 2-fold in Fpat $^{-1-}$ mice (Figure 4A). The rates of BrdU incorporation into hepatocytes during a 72-h period in Wy-treated mice, compared with vehicle-treated mice, increased 10-fold in both the control and ppat $^{-/-}$mice (Figure 4B). Thus, GPAT1 was not critical for PPARa-mediated induction of hepatocellular proliferation.

\section{Discussion}

The development of HCC is often preceded by hepatitis C infection, hepatitis B infection, aflatoxin B1 intoxication, or NASH. NASH develops in association with a high-fat diet and obesity. With the dramatic worldwide increase in the incidence of obesity and NASH, the WHO has predicted that as many as 2 million of the currently obese individuals will develop cirrhosis that may progress to HCC (Schreuder et al. 2008). Although HCC develops as part of the natural progression of NASH, the role of complex lipid synthesis in HCC development remains unresolved. In patients with chronic hepatitis $\mathrm{C}$ infection, hepatic steatosis is an independent risk factor (Ohata et al. 2003), likely due to the requirement of lipid droplets for viral propagation (Herker and Ott 2011). Hepatic steatosis increases the risk of HCC in several mouse models. For instance, the typically HCC-resistant C57B1/6 mice develop NASH and HCC when fed a high (60\%) fat diet (Hill-Baskin et al. 2009), and after HCC induction with a high dose of DEN, both dietary and genetic obesity enhance hepatic tumorigenesis in C57B1/6 mice (Park et al. 2010). In another model, hepatic steatosis induced by a choline-deficient diet plus methionine in the drinking water or induced by a choline-deficient L-amino acid-defined diet caused HCC in 100\% of mice within one year of beginning the diet (Fausto and Campbell 2010). These data show a strong link between diet, fatty liver, and risk of liver tumorigenesis.

Another mouse model of hepatic steatosis is the hepatocyte-specific deficiency of the phosphatase and tensin homolog (PTEN), an inhibitor of PI3 kinase. These $P T E N^{-1}$ mice 
have activated Akt (serine/threonine protein kinase) and MAPK (mitogen-activated protein kinase), steatohepatitis, and an increase in hepatocellular carcinomas (Horie et al. 2004; Watanabe et al. 2007). In mice that overexpress platelet-derived growth factor-C (PDGF-C) (Campbell et al. 2005), 80\% develop hepatic steatosis that progresses to fibrosis and stellate cell activation; activated stellate cells produce matrix and factors that stimulate hepatocytes to proliferate and generate chemokines that attract inflammatory cells (Jou, Choi, and Diehl 2008). By 12 months, the hepatosteatotic PDGF-C transgenic mice develop HCC. Like $P T E N^{-1-}$ mice, the PDGF-C transgenic mice also have activated growth factor-mediated signaling pathways that include extracellular-regulated kinases-1 and -2 (ERK1 and 2) and AKT. The overexpression of another growth factor, fibroblast growth factor 21 (Fgf21) in the liver attenuates DEN-induced HCC (Huang, Yu, Jin, Yang, et al. 2006), suggesting that not all growth factor-stimulated pathways increase HCC development. Conversely, the liverspecific overexpression of the fibroblast growth factor receptor 1 (FGFR1) increases DENinduced HCC (Huang, Yu, Jin, Kobayashi, et al. 2006), although FGFR1 knockout mice are not protected from DEN-initiated HCC (Huang, Yu, Jin, Yang, et al. 2006), suggesting that this signaling pathway can influence, but is not required for DEN-initiated HCC. Taken as a whole, these data suggest that HCC development is associated with specific growth factors that stimulate hepatocyte proliferation in the presence of obesity and hepatic steatosis.

Consistent with the role of GPAT in initiating the synthesis of TAG, compared with wildtype mice, Fpat $^{-1-}$ mice synthesize and store less hepatic TAG and shunt more fatty acid toward oxidation (Hammond et al. 2005). Thus, after Fpat $^{-1-}$ mice were fed a high-fat diet for 4 months, their livers contained 60\% less fat than those of wildtype controls (Hammond et al. 2005); and, compared with $o b / o b$ mice, double knockout $o b / o b-G p a t^{/-}$mice have a $59 \%$ reduction in hepatic TAG content (Wendel et al. 2010). Compared with controls, the content of acyl-CoA is higher in Fpat $^{-/-}$liver, whereas liver content of the signaling lipids, lysophosphatidic acid, and diacylglycerol, is lower (Neschen et al. 2005). Because GPAT1, which acylates the $s n-1$ position of glycerolipids, has a strong preference for saturated fatty acyl-CoAs, particularly palmitoyl-CoA (Coleman and Lee 2004), the content of palmitate in Gpat $^{-/-}$liver phosphatidylcholine and phosphatidylethanolamine is diminished at the $s n-1$ position (Hammond et al. 2002). As a result of the decrease in palmitate, these phospholipids contain relatively more 18:1 (oleate) at the $s n-1$ position and more 20:4 46 (arachidonate) at the $s n-2$ position. Gpat $^{-1-}$ livers contain more 4-hydroxy-2-nonenal, a deleterious product of 20:4 46 peroxidation that occurs with oxidative stress, as well as increased rates of hepatocyte apoptosis and proliferation (Hammond et al. 2007). Thus, Gpat $^{-1-}$ livers exhibit both reduced synthesis of TAG and an increased rate of cell turnover.

In mice, PPARa up-regulates genes that enhance hepatocyte proliferation; the resulting selective clonal expansion may lead to tumorigenesis (Klaunig et al. 2003). Although PPAR transcriptional activity regulates genes of lipid metabolism, we failed to find a requirement for GPAT1-mediated lipid synthesis and PPARa agonist-mediated hepatocyte proliferation. Hepatitis C-induction of HCC in mice is enhanced by liver-specific overexpression of PPARa and by exposure to PPARa agonists, suggesting that PPARa is critical for hepatitis C-induction of HCC (Tanaka et al. 2008). Our data, however, suggest that glycerolipid synthesis via the Kennedy pathway does not alter PPARa-mediated hepatocyte proliferation. Because chronically activated PPARa results in hepatic tumors in rodents, endogenous ligands like long-chain fatty acids or their metabolitesmight contribute to hepatic carcinogenesis (Yu, Rao, and Reddy 2003), but this is unlikely to occur in Gpat1 $^{-1-}$ mice. Acyl-CoA content is 64\% higher in livers with deficient GPAT1 (Neschen et al. 2005); thus, Fpat $^{-1-}$ livers might have increased acyl-CoAs that enter mitochondrial or extra-mitochondrial pathways that increase oxidative stress and thereby alter either gene expression or DNA itself (Ockner, Kaikaus, and Bass 1993). In fact, livers from Gpat $1^{-/-}$ 
mice do exhibit increased oxidative stress (Hammond et al. 2007) although they proved to be less susceptible to chemically-induced tumorigenesis.

Compared to wildtype C3H mice, Gpat1 $1^{-1-}$ mice had fewer DEN-induced hepatic foci, adenomas, and HCC. At 21 and 34 weeks, Gpat $^{-1-}$ livers contained fewer preneoplastic foci and tumors. This decreased susceptibility suggests that the absence of GPAT1 causes metabolic and biochemical alterations in mouse livers that diminish its carcinogenic risk. These alterations may include the propensity of $\mathrm{Gpat}^{-{ }^{--}}$mice to resist hepatic steatosis in both diet and genetic models (Hammond et al. 2005; Wendel et al. 2010). Mice deficient in GPAT1 were protected from HCC despite increases in both proliferation and measures of oxidative stress (Hammond et al. 2007). As was shown in Gpat $^{-1-}$ livers on the C57Bl/6 background, the absence of GPAT1 in the $\mathrm{C} 3 \mathrm{H}$ strain resulted in a 2-fold greater $\mathrm{BrdU}$ incorporation than occurred in wildtype $\mathrm{C} 3 \mathrm{H}$ livers. In a comparison of $\mathrm{C} 3 \mathrm{H}$ and $\mathrm{C} 3 \mathrm{~B} 6 \mathrm{~F} 1$ $(\mathrm{C} 3 \mathrm{H} \times \mathrm{C} 57 \mathrm{BL} / 6)$ mice in which tumors were induced with a regimen of $16 \mathrm{mg} / \mathrm{kg}$ DEN plus 500 p.p.m. sodium phenobarbital in drinking water and examined at 23 weeks, labeling with BrdU increased in foci and adenomas; however, in unaffected or normal hepatocytes, the level of BrdU incorporation did not correlate with the greatly increased number of tumors in the C3H mice (Goldsworthy and Fransson-Steen 2002). Thus in Gpat1 $^{-1-}$ liver, the increase in ROS, the impaired mitochondrial function, and the increase in apoptosis may diminish the propensity to develop liver tumors.

Exposure to the PPARa ligand WY-14,643 had similar effects on liver weight and BrdU incorporation in control and Gpat $^{-1-}$ liver, indicating that at baseline, the two genotypes did not differ in their capacity for PPARa-mediated stimulation. Further, in both genotypes, mRNA abundance is similar for PPARa itself, for peroxisome proliferator-activated receptor-a coactivator-1, and for the PPARa target genes, acyl-CoA oxidase, carnitine palmitoyltransferase-1, and long-chain acyl-CoA dehydrogenase (Hammond et al. 2005).

The toxicity associated with hepatic steatosis has been associated with excess fatty acid metabolites that harm hepatocytes and render them unable to regenerate properly (Jou, Choi, and Diehl 2008). However, unlike models in which steatosis and oxidative stress lead to cell proliferation and HCC, Gpat $^{-1-}$ mice are resistant to both diet-induced and genetic steatosis, but have up-regulated fatty acid oxidation, and an increased rate of hepatocyte proliferation (Hammond et al., 2007, Wendel et al., 2010) that is associated with reduced susceptibility to tumorigenic foci and adenomas. It is as yet unclear as to whether the increase in hepatocyte turnover or the lack of a toxic lipid metabolite protected the Gpat $^{-1-}$ mice from tumorigenesis. This relative protection could provide clues as to the underlying pathogenesis of HCC.

\section{Acknowledgments}

We greatly appreciate the review of the article by Drs. Mark Hoenerhoff, Gordon Flake, and Grace Kissling of NIEHS, as well as the statistical analysis by Dr. Kissling. We thank Eli Ney for her assistance with photomicroscopy.

This work was supported by NIH grant DK056598 (RAC); a predoctoral training grant HL069768 in Integrative Vascular Biology (JME); P30 DK034987, P30-ES010126, P30 DK056350, and a predoctoral fellowship from the American Heart Association-Mid-Atlantic Region (JME). This research was also supported in part by the Intramural Research Program of the NIH, National Institute of Environmental Health Sciences.

\section{Abbreviations}

DEN diethylnitrosamine 


$\begin{array}{ll}\text { GPAT } & \text { glycerol-3-phosphate acyltransferase } \\ \text { HCC } & \text { hepatocellular carcinoma } \\ \text { HCA } & \text { hepatocellular adenoma } \\ \text { NAFLD } & \text { non-alcoholic fatty liver disease } \\ \text { NASH } & \text { non-alcoholic steatohepatitis } \\ \text { PPAR } & \text { peroxisome-proliferator activated receptor } \\ \text { ROS } & \text { reactive oxygen species } \\ \text { TAG } & \text { triacylglycerol }\end{array}$

\section{References}

Bogacka I, Ukropcova B, McNeil M, Gimble JM, Smith SR. Structural and functional consequences of mitochondrial biogenesis in human adipocytes in vitro. J Clin Endocrinol Metab. 2005; 90:66506656. [PubMed: 16204368]

Bosch FX, Ribes J, Diaz M, Cleries R. Primary liver cancer: worldwide incidence and trends. Gastroenterology. 2004; 127:S5-S16. [PubMed: 15508102]

Campbell JS, Hughes SD, Gilbertson DG, Palmer TE, Holdren MS, Haran AC, Odell MM, Bauer RL, Ren HP, Haugen HS, Yeh MM, Fausto N. Platelet-derived growth factor C induces liver fibrosis, steatosis, and hepatocellular carcinoma. Proc Natl Acad Sci U. S. A. 2005; 102:3389-3394. [PubMed: 15728360]

Cao J, Li JL, Li D, Tobin JF, Gimeno RE. Molecular identification of microsomal acyl-CoA: glycerol-3-phosphate acyltransferase, a key enzyme in de novo triacylglycerol synthesis. Proc Natl Acad Sci U. S. A. 2006; 103:19695-19700. [PubMed: 17170135]

Chang Y-Y, Kennedy EP. Biosynthesis of phosphatidyl glycerophosphate in Escherichia coli. J Lipid Res. 1967; 8:447-455. [PubMed: 4860577]

Chen Y, Kuo MS, Li S, Bui HH, Peake DA, Sanders PE, Thibodeaux SJ, Chu S, Qian YW, Zhao Y, Bredt DS, Moller DE, Konrad RJ, Beigneux AP, Young SG, Cao G. AGPAT6 is a novel microsomal glycerol-3-phosphate acyltransferase (GPAT). J Biol Chem. 2008; 283:10048-10057. [PubMed: 18238778]

Coleman RA, Lee DP. Enzymes of triacylglycerol synthesis and their regulation. Prog Lipid Res. 2004; 43:134-176. [PubMed: 14654091]

Das SK, Haldar D. The differential effect of polymyxin B1 on guinea pig lung mitochondrial and microsomal glycerophosphate acyltransferase. Lipids. 1987; 22:757-759. [PubMed: 2828812]

Diwan BA, Rice JM, Ward JM. Strain-dependent effects of phenobarbital on liver tumor promotion in inbred mice. Prog Clin Biol Res. 1990; 331:69-83. [PubMed: 2179966]

Drinkwater NR, Ginsler JJ. Genetic control of hepatocarcinogenesis in C57BL/6 J and C3H/HeJ inbred mice. Carcinogenesis. 1986; 7:1701-1707. [PubMed: 3757172]

El-Serag HB, Mason AC. Rising incidence of hepatocellular carcinoma in the United States. N Engl J Med. 1999; 340:745-750. [PubMed: 10072408]

Fausto N, Campbell JS. Mouse models of hepatocellular carcinoma. Semin Liver Dis. 2010; 30:87-98. [PubMed: 20175036]

Goldsworthy TL, Fransson-Steen R. Quantitation of the cancer process in C57BL/6 J, B6C3F1 and C3H/HeJ mice. Toxicol Pathol. 2002; 30:97-105. [PubMed: 11890483]

Gonzalez FJ, Shah YM. PPARalpha: mechanism of species differences and hepatocarcinogenesis of peroxisome proliferators. Toxicology. 2008; 246:2-8. [PubMed: 18006136]

Hammond LE, Albright CD, He L, Rusyn I, Watkins SW, Doughman SD, Lemasters JJ, Coleman RA. Increased oxidative stress is associated with balanced increases in hepatocyte apoptosis and proliferation in glycerol-3-phosphate acyltransferase-1 deficient mice. Exper Molec Pathol. 2007; 82:210-219. [PubMed: 17258706] 
Hammond LE, Gallagher PA, Wang S, Posey-Marcos E, Hiller S, Kluckman K, Maeda N, Coleman RA. Mitochondrial glycerol-3-phosphate acyltransferase-deficient mice have reduced weight and liver triacylglycerol content and altered glycerolipid fatty acid composition. Mol Cell Biol. 2002; 22:8204-8214. [PubMed: 12417724]

Hammond LE, Neschen S, Romanelli AJ, Cline GW, Ilkayeva OR, Shulman GI, Muoio DM, Coleman RA. Mitochondrial glycerol-3-phosphate acyltransferase-1 is essential in liver for the metabolism of excess acyl-CoAs. J Biol Chem. 2005; 280:25629-25636. [PubMed: 15878874]

Harada, T.; Enomoto, A.; Boorman, GA.; Maronpot, RR. Liver and gall bladder. In: Maronpot, RR., editor. Pathology of the Mouse. Vienna, IL: Cache River Press; 1999. p. 140-151.

Herker E, Ott M. Unique ties between hepatitis $\mathrm{C}$ virus replication and intracellular lipids. Trends Endocrinol Metab. 2011; 22:241-248. [PubMed: 21497514]

Hill-Baskin AE, Markiewski MM, Buchner DA, Shao H, DeSantis D, Hsiao G, Subramaniam S, Berger NA, Croniger C, Lambris JD, Nadeau JH. Diet-induced hepatocellular carcinoma in genetically predisposed mice. Hum Mol Genet. 2009; 18:2975-2988. [PubMed: 19454484]

Horie Y, Suzuki A, Kataoka E, Sasaki T, Hamada K, Sasaki J, Mizuno K, Hasegawa G, Kishimoto H, Iizuka M, Naito M, Enomoto K, Watanabe S, Mak TW, Nakano T. Hepatocyte-specific Pten deficiency results in steatohepatitis and hepatocellular carcinomas. J Clin Invest. 2004; 113:17741783. [PubMed: 15199412]

Huang X, Yu C, Jin C, Kobayashi M, Bowles CA, Wang F, McKeehan WL. Ectopic activity of fibroblast growth factor receptor 1 in hepatocytes accelerates hepatocarcinogenesis by driving proliferation and vascular endothelial growth factor-induced angiogenesis. Cancer Res. 2006; 66:1481-1490. [PubMed: 16452204]

Huang X, Yu C, Jin C, Yang C, Xie R, Cao D, Wang F, McKeehan WL. Forced expression of hepatocyte-specific fibroblast growth factor 21 delays initiation of chemically induced hepatocarcinogenesis. Mol Carcinog. 2006; 45:934-942. [PubMed: 16929488]

Jou J, Choi SS, Diehl AM. Mechanisms of disease progression in nonalcoholic fatty liver disease. Semin Liver Dis. 2008; 28:370-379. [PubMed: 18956293]

Kennedy EP. Biosynthesis of complex lipids. Fed Proc. 1961; 20:934-940. [PubMed: 14455159]

Klaunig JE, Babich MA, Baetcke KP, Cook JC, Corton JC, David RM, DeLuca JG, Lai DY, McKee RH, Peters JM, Roberts RA, Fenner-Crisp PA. PPARalpha agonistinduced rodent tumors: modes of action and human relevance. Crit Rev Toxicol. 2003; 33:655-780. [PubMed: 14727734]

Lewin TM, Schwerbrock NMJ, Lee DP, Coleman RA. Identification of a new glycerol-3-phosphate acyltransferase isoenzyme, mtGPAT2, in mitochondria. J Biol Chem. 2004; 279:13488-13495. [PubMed: 14724270]

Marsman DS, Popp JA. Biological potential of basophilic hepatocellular foci and hepatic adenoma induced by the peroxisome proliferator, Wy-14,643. Carcinogenesis. 1994; 15:111-117. [PubMed: 7507413]

Nagle CA, Verges L, Wang S, deJong H, Wang S, Lewin TM, Reue K, Coleman RA. Identification of a novel sn-glycerol-3-phosphate acyltransferase isoform, GPAT4 as the enzyme deficient in Agpat6-/- mice. J Lipid Res. 2008; 49:823-831. [PubMed: 18192653]

Neschen S, Morino K, Hammond LE, Zhang D, Liu ZX, Romanelli AJ, Cline GW, Pongratz RL, Zhang XM, Choi CS, Coleman RA, Shulman GI. Prevention of hepatic steatosis and hepatic insulin resistance in mitochondrial acyl-CoA: glycerol-sn-3-phosphate acyltransferase 1 knock out mice. Cell Metab. 2005; 2:55-65. [PubMed: 16054099]

Neuschwander-Tetri BA, Caldwell SH. Nonalcoholic steatohepatitis: summary of an AASLD single topic conference. Hepatology. 2003; 37:1202-1219. [PubMed: 12717402]

Ockner RK, Kaikaus RM, Bass NM. Fatty-acid metabolism and the pathogenesis of hepatocellular carcinoma: review and hypothesis. Hepatology. 1993; 18:669-676. [PubMed: 8395460]

Ohata K, Hamasaki K, Toriyama K, Matsumoto K, Saeki A, Yanagi K, Abiru S, Nakagawa Y, Shigeno M, Miyazoe S, Ichikawa T, Ishikawa H, Nakao K, Eguchi K. Hepatic steatosis is a risk factor for hepatocellular carcinoma in patients with chronic hepatitis $\mathrm{C}$ virus infection. Cancer. 2003; 97:3036-3043. [PubMed: 12784339] 
Park EJ, Lee JH, Yu GY, He G, Ali SR, Holzer RG, Osterreicher CH, Takahashi H, Karin M. Dietary and genetic obesity promote liver inflammation and tumorigenesis by enhancing IL-6 and TNF expression. Cell. 2010; 140:197-208. [PubMed: 20141834]

Parkin DM, Bray F, Ferlay J, Pisani P. Global cancer statistics, 2002. CA Cancer J Clin. 2005; 55:74108. [PubMed: 15761078]

Pillai AA, Rinella ME. Non-alcoholic fatty liver disease: is bariatric surgery the answer? Clin Liver Dis. 2009; 13:689-710. [PubMed: 19818313]

Reznik-Schuller HM. Pathogenesis of tumors induced with Nnitrosomethylpiperazine in the olfactory region of the rat nasal cavity. J Natl Cancer Inst. 1983; 71:165-172. [PubMed: 6191067]

Schattenberg JM, Schuchmann M, Galle PR. Cell death and hepatocarcinogenesis: Dysregulation of apoptosis signaling pathways. Journal of Gastroenterology and Hepatology. 2011; 26(Suppl 1): 213-219. [PubMed: 21199533]

Schlossman DM, Bell RM. Microsomal sn-glycerol 3-phosphate and dihydroxyacetone phosphate acyltransferase activities from liver and other tissues. Evidence for a single enzyme catalizing both reactions. Arch Biochem Biophys. 1977; 182:732-742. [PubMed: 20060]

Schreuder TC, Verwer BJ, van Nieuwkerk CM, Mulder CJ. Nonalcoholic fatty liver disease: an overview of current insights in pathogenesis, diagnosis and treatment. World J Gastroenterol. 2008; 14:2474-2486. [PubMed: 18442193]

Takahashi M, Dinse GE, Foley JF, Hardisty JF, Maronpot RR. Comparative prevalence, multiplicity, and progression of spontaneous and vinyl carbamate-induced liver lesions in five strains of male mice. Toxicol Pathol. 2002; 30:599-605. [PubMed: 12371669]

Tanaka N, Moriya K, Kiyosawa K, Koike K, Gonzalez FJ, Aoyama T. PPARalpha activation is essential for $\mathrm{HCV}$ core protein-induced hepatic steatosis and hepatocellular carcinoma in mice. $\mathrm{J}$ Clin Invest. 2008; 118:683-694. [PubMed: 18188449]

Watanabe S, Horie Y, Kataoka E, Sato W, Dohmen T, Ohshima S, Goto T, Suzuki A. Non-alcoholic steatohepatitis and hepatocellular carcinoma: lessons from hepatocyte-specific phosphatase and tensin homolog (PTEN)-deficient mice. Journal of Gastroenterology and Hepatology. 2007; 22(Suppl 1):S96-S100. [PubMed: 17567478]

Wendel AA, Li LO, Li Y, Cline GW, Shulman GI, Coleman RA. Glycerol-3-phosphate acyltransferase 1 deficiency in ob/ob mice diminishes hepatic steatosis but does not protect against insulin resistance or obesity. Diabetes. 2010; 59:1321-1329. [PubMed: 20200319]

World Health Organization. Mortality Database. Vol. Vol. 2009. Geneva, Switzerland: 2009.

Yu S, Rao S, Reddy JK. Peroxisome proliferator-activated receptors, fatty acid oxidation, steatohepatitis and hepatocarcinogenesis. Curr Mol Med. 2003; 3:561-572. [PubMed: 14527087] 

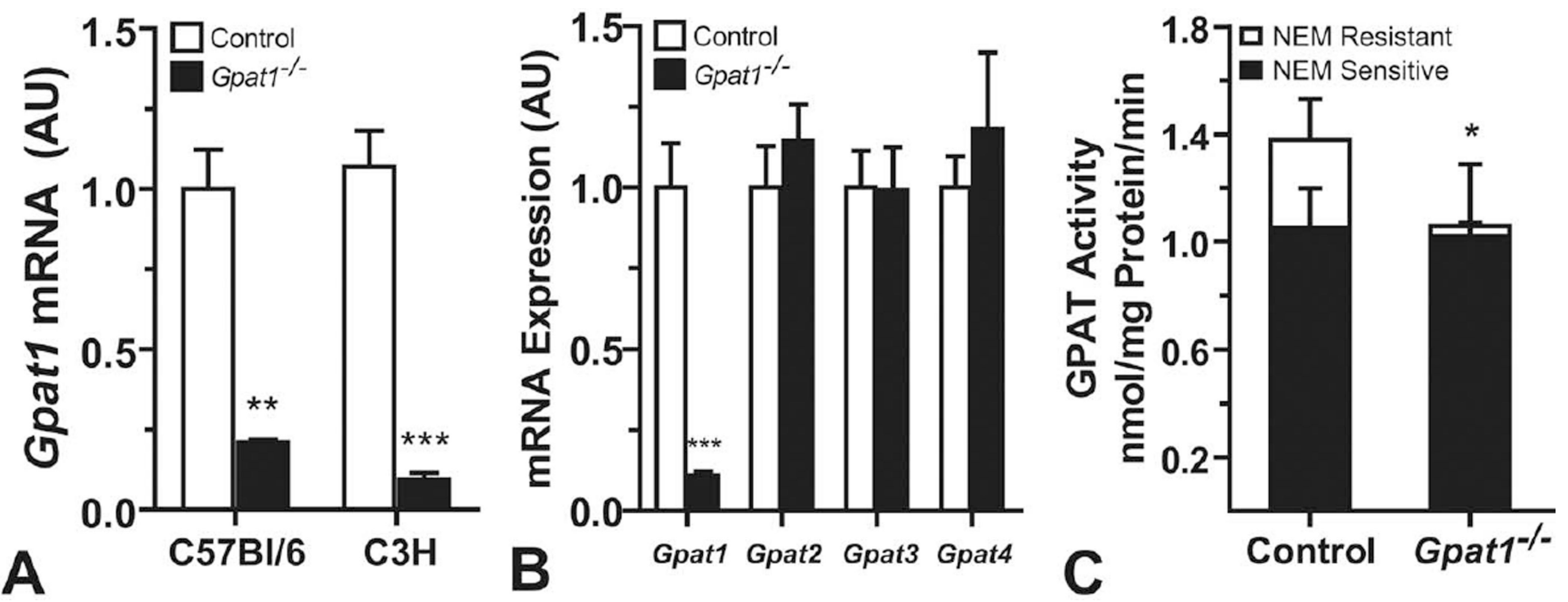

FIGURE 1.

Gpat $^{-1-}$ mice on the $\mathrm{C} 3 \mathrm{H}$ strain background had decreased Gpat1 expression and activity in liver. (A) Gpat1 mRNA abundance in $\mathrm{C} 57 \mathrm{Bl} / 6$ and $\mathrm{C} 3 \mathrm{H}$ control livers. (B) Gpat2, Gpat3, and $G$ pat4 mRNA abundance in control and $G$ pat1 ${ }^{-1-}$ livers on the $\mathrm{C} 3 \mathrm{H}$ background. (C) GPAT-specific activity in control and ppat $^{-/-}$livers on the $\mathrm{C} 3 \mathrm{H}$ background. Data represent the average $\pm \mathrm{SEM} ; * p \leq .05, * * p \leq .01, * * * p \leq .001$, between genotypes. 

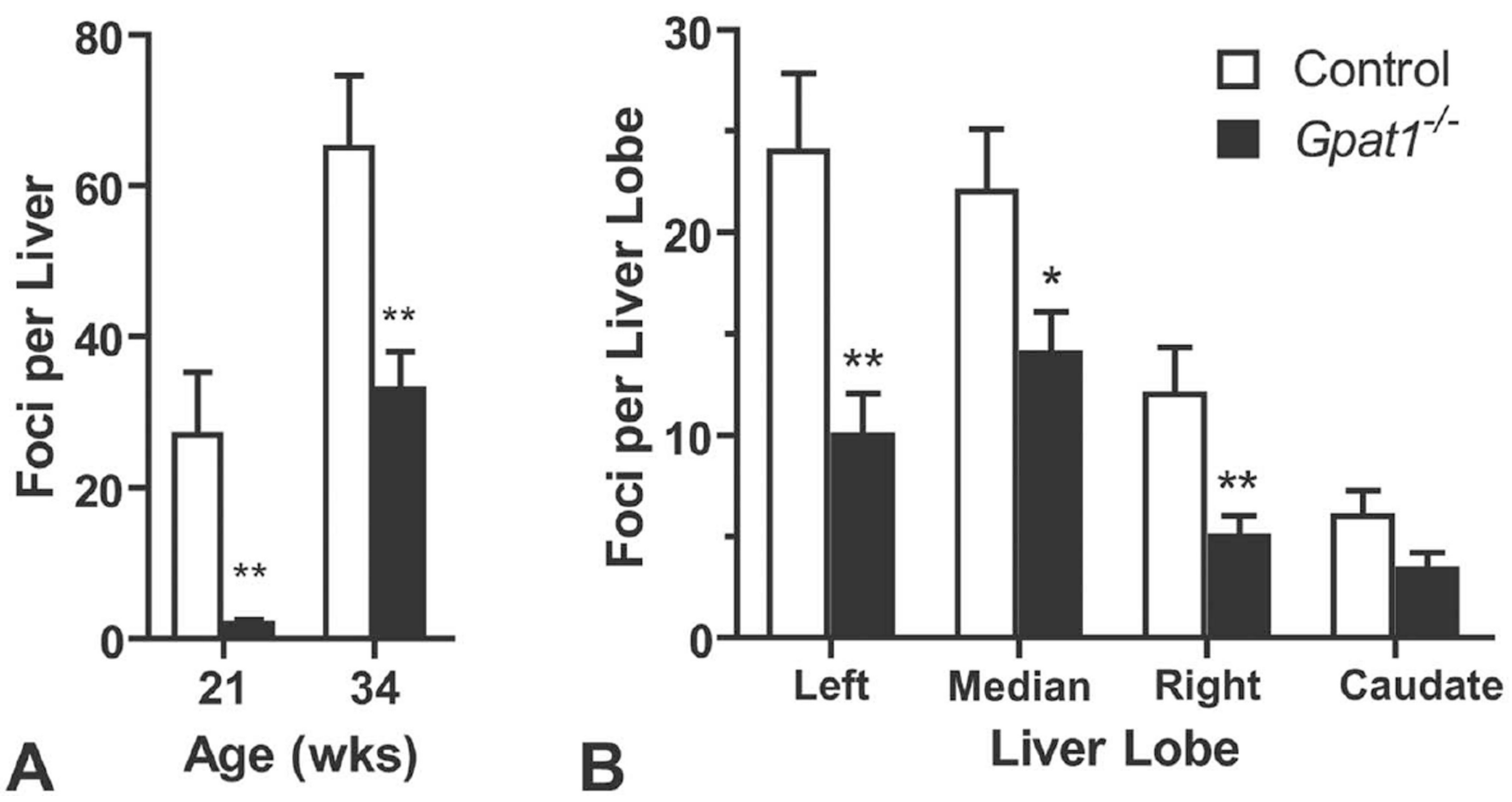

FIGURE 2.

Gpat $1^{-/-}$mice had reduced susceptibility to liver cancer. (A) Average number of macroscopically visible liver nodules in control and $\mathrm{Gpat}^{-1-}$ 21-week-old mice (control, $n$ $=12 ;$ Gpat1 $^{-1-}, n=11$ ) and 34-week-old mice (control, $n=15 ;$ Gpat1 $\left.^{-/-}, n=19\right)$. (B) Average of macroscopically visible nodules per liver lobe at $34 \mathrm{wk}$ of age $(n=15)$. Data represent the average $\pm \mathrm{SEM} ; * p \leq .05, * * p \leq .01$, between genotypes. 

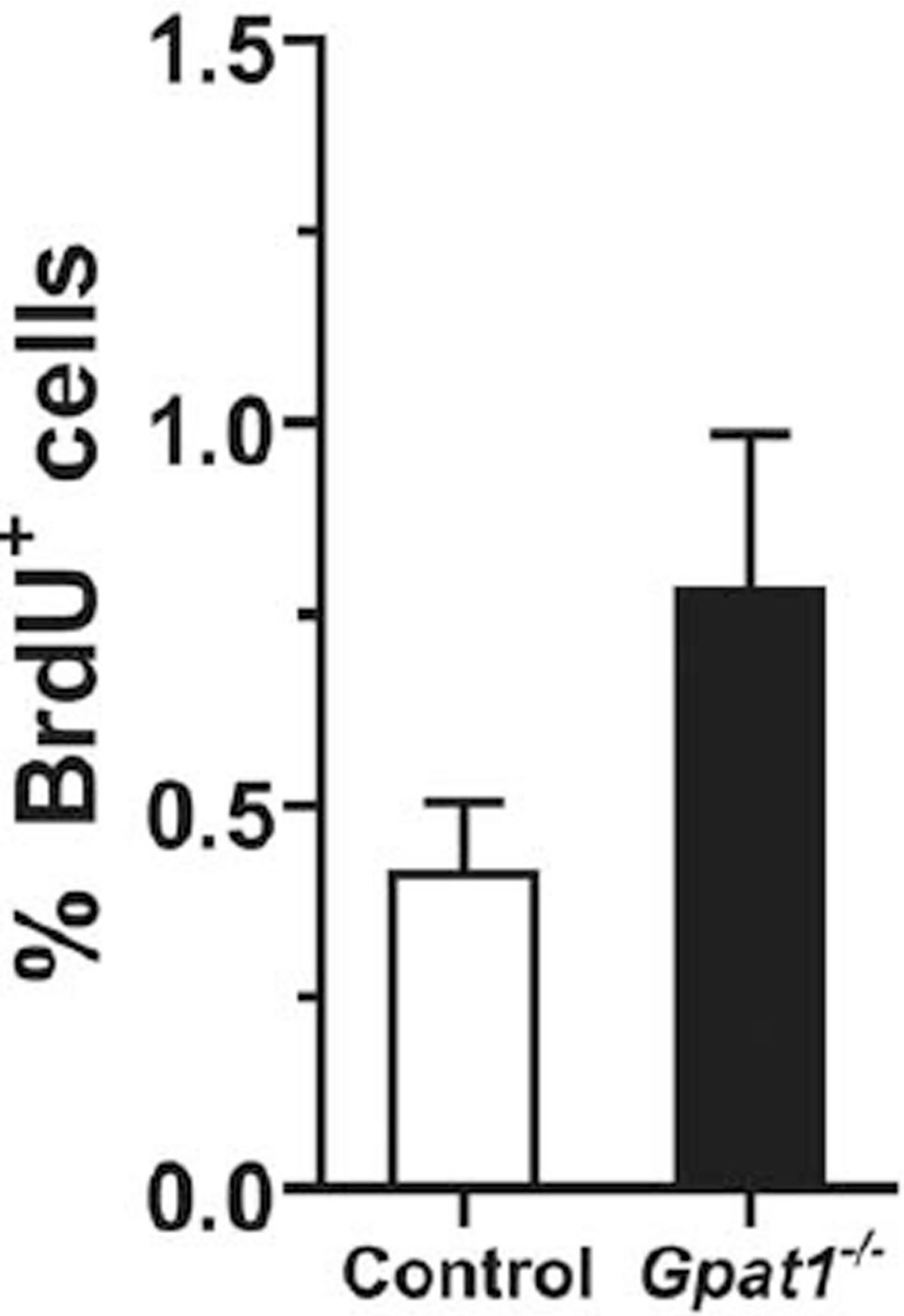

$+$
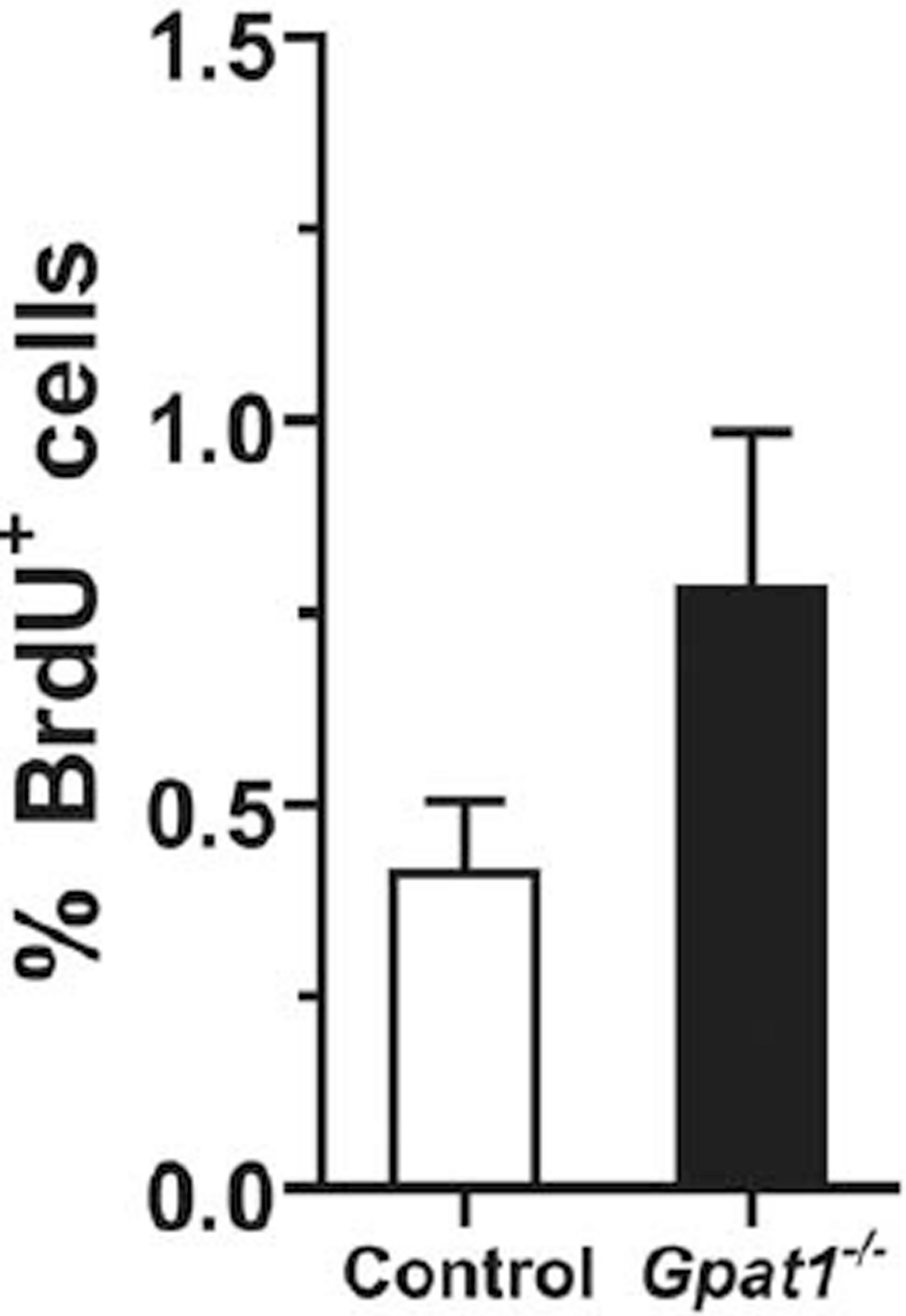

FIGURE 3.

Gpat $^{-1-}$ livers from 8-week-old, non-DEN-exposed mice had increased rates of hepatocyte proliferation. Percentage of hepatocytes positive for BrdU incorporation after a 48-h exposure. 

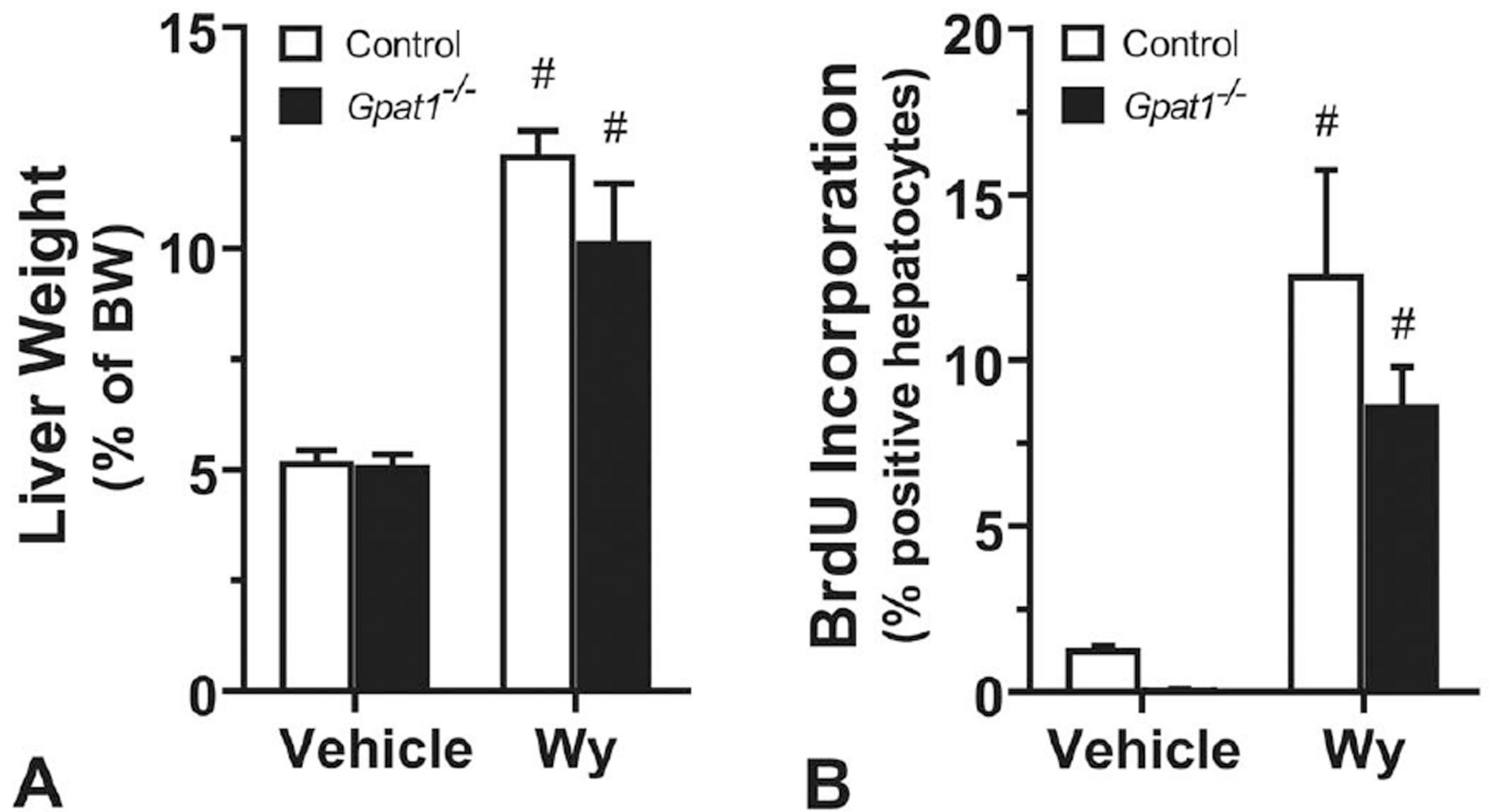

FIGURE 4.

Gpat $^{-1-}$ and control hepatocytes increased BrdU incorporation similarly in response to Wy-14643. (A) Liver weight, as a percentage of body weight; and (B) BrdU incorporation into hepatocytes in control and $\mathrm{Gpat}^{-1-}$ mice with or without (No Trt) $1 \mathrm{wk}$ of exposure to Wy-14643, $n=3$. Data represent the average \pm SEM; \#, $p \leq .05$ between no treatment and Wy-14643-treated mice within the genotype. 

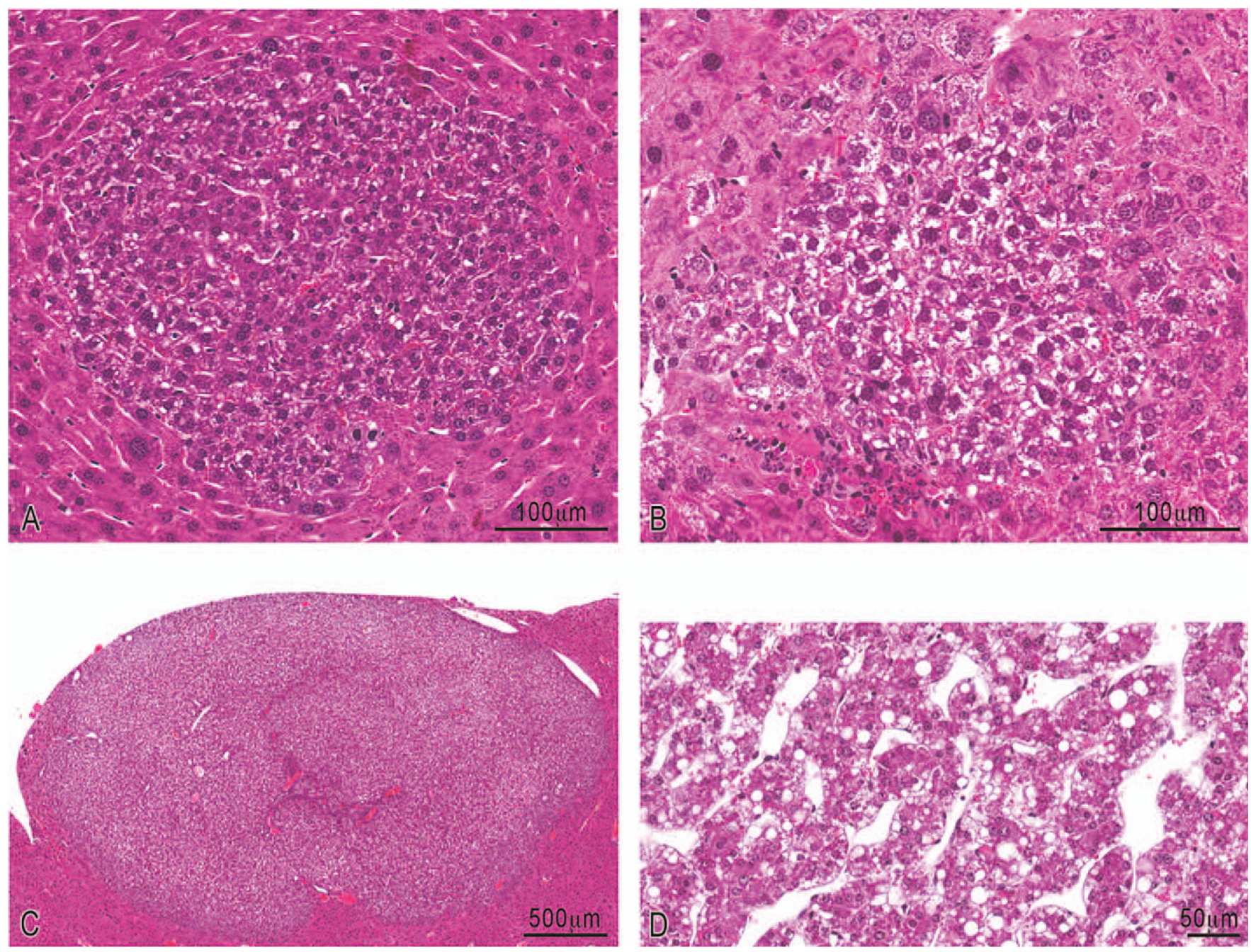

FIGURE 5.

Representative photomicrographs of lesions in $\mathrm{C} 3 \mathrm{H}$ livers. (A) Hematoxylin and eosinstained basophilic focus of altered hepatocytes; (B) clear cell focus of altered hepatocytes; (C) low magnification of an hepatocellular adenoma; (D) high magnification of an hepatocellular carcinoma characterized by pleomorphic hepatocytes forming irregular trabeculae that are often three cells or greater in width. 


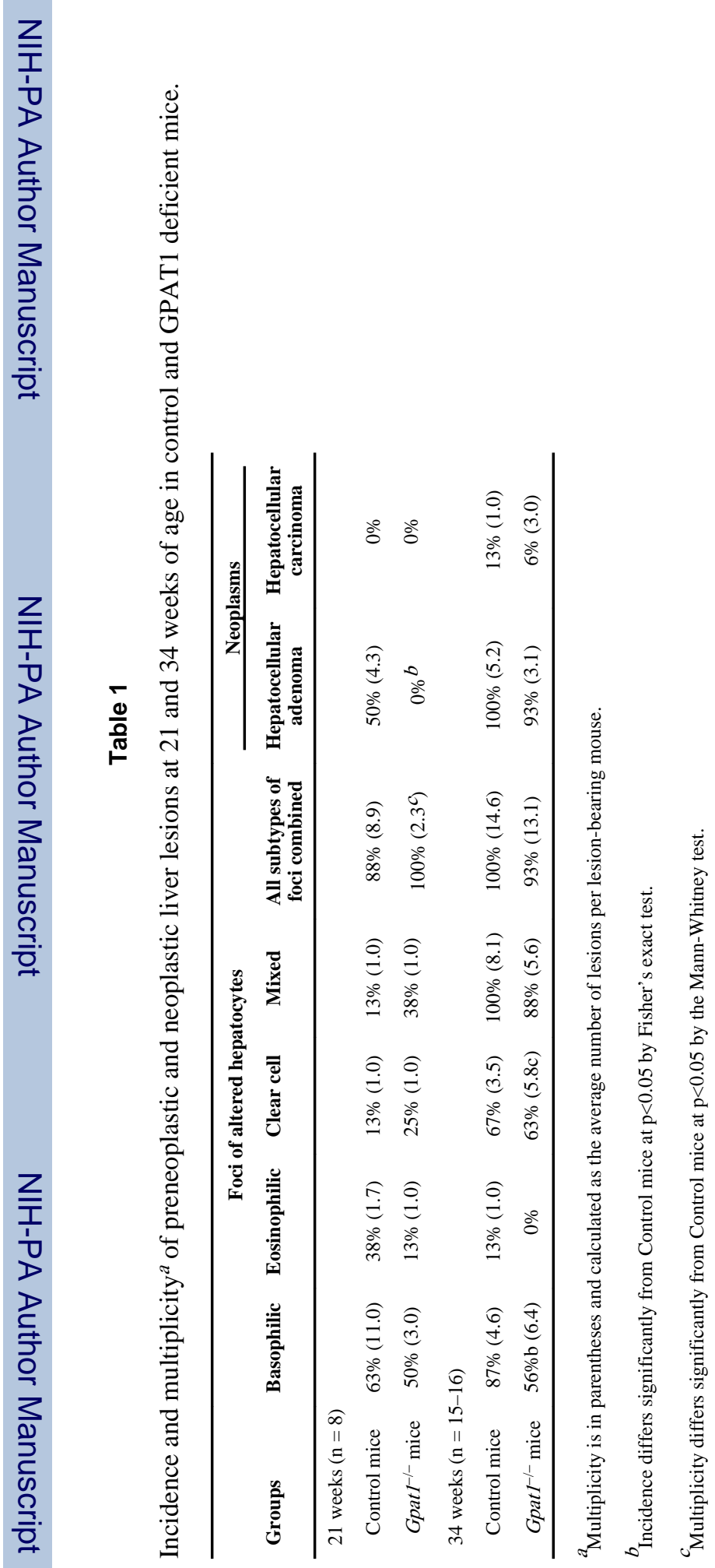

